\title{
DESDE ALMA-ATA AL CIUDADANO DIGITAL: HACIA UNA ATENCIÓN PRIMARIA EN SALUD DIGITALIZADA EN EL PERÚ
}

\author{
Walter H. Curioso 1,a, Milagro Henríquez-Suarez 2,b, Elizabeth Espinoza-Portilla ${ }^{3, c}$
}

\begin{abstract}
RESUMEN
Numerosos cambios vienen experimentando los países de la Región de las Américas luego de la Declaración de AlmaAta en 1978, como por ejemplo la globalización, nuevos perfiles epidemiológicos, demográficos, y la revolución de las tecnologías de información y comunicación. Sin embargo, los modelos de atención primaria en salud en muchos países de la región persisten con numerosos retos, incluyendo la desarticulación de los niveles de atención y una gestión de la información poco eficiente y oportuna. Urge implementar un sistema de información en salud robusto, flexible e interoperable que permita el acceso oportuno a información precisa, confiable, y consistente acerca de la salud de las personas y de la población. En este artículo se discuten las perspectivas para desarrollar una atención primaria en salud digitalizada.
\end{abstract}

Palabras clave: Sistemas de información en salud; Informática médica; Telesalud; Tecnologías de información (fuente: DeCS BIREME).

\section{FROM ALMA-ATA TO THE DIGITAL CITIZEN: TOWARDS A DIGITAL PRIMARY HEALTH CARE IN PERU}

\begin{abstract}
The countries of the Region of the Americas Numerous have experienced many changes since the Declaration of AlmaAta in 1978, such as globalization, new epidemiologic and demographic profiles, and the information and communication technology revolution. Nevertheless, the primary health care models in many countries of the region still endure numerous challenges, including the disarticulation of the levels of care and an inefficient management of information. It is urgent to implement a strong, flexible, and interoperable health information system that would allow the suitable access to precise, reliable, and consistent information about the health of the population. This article discusses the perspectives to develop a digital primary health care system.
\end{abstract}

Keywords: Health information systems; Medical informatics; Telehealth; Information technology (source: MeSH NLM).

\section{EL FORTALECIMIENTO DE LA ATENCIÓN PRIMARIA EN SALUD EN LAS AMÉRICAS DESPUÉS DE ALMA-ATA UTILIZANDO LAS TECNOLOGÍAS}

La atención primaria en salud forma parte del sistema nacional de salud, del que constituye la función central y su núcleo principal, como del desarrollo social y económico global de la comunidad (1). Las ideas fundamentales de la Declaración de Alma-Ata de 1978, que difundió varios conceptos innovadores en salud pública, siguen siendo válidas y deben ser integradas en los sistemas sanitarios de la Región de las Américas para fortalecer la atención primaria en salud (2).

En el 2005, uno de los compromisos establecidos por los países de la Región de las Américas en la Declaración Regional sobre las nuevas orientaciones de la atención

\footnotetext{
Department of Biomedical Informatics and Medical Education, School of Medicine, University of Washington. Seattle, Washington, EE. UU.

Facultad de Ciencias Sociales, Universidad Nacional José Faustino Sánchez Carrión. Huacho, Perú.

Instituto de Gobierno y de Gestión Pública. Universidad de San Martín de Porres. Lima, Perú.

Médico cirujano, magíster en Salud Pública y doctor en Informática Biomédica; ${ }^{\text {b }}$ Licenciada en Trabajo Social, magíster en Salud Pública y doctora en Administración; ${ }^{\mathrm{c}}$ Médico cirujano, magíster en Estudios de Políticas Públicas, magíster en Demografía y Población, y doctora en Gobierno y Políticas Públicas.

Recibido: 04/06/2018 Aprobado: 29/08/2018 En línea: 21/12/2018
}

Citar como: Curioso WH, Henríquez-Suarez M, Espinoza-Portilla E. Desde Alma-Ata al ciudadano digital: hacia una atención primaria en salud digitalizada en el Perú. Reporte de un caso. Rev Peru Med Exp Salud Publica. 2018;35(4):678-83.doi:10.17843/rpmesp.2018.354.3710. 
primaria de salud (Declaración de Montevideo) fue el desarrollo de la tecnología como componente fundamental de una estrategia para renovar y fortalecer la atención primaria de salud ${ }^{(2)}$. Asimismo, se estableció que la atención primaria en salud desempeña un papel importante en la coordinación «de la continuidad de la atención y del flujo de información a lo largo de todo el sistema de atención de salud» (2).

Los servicios de primer nivel de atención enfrentan nuevos desafíos sanitarios ${ }^{(3)}$ y por si solos no son capaces para responder de manera apropiada a las necesidades de atención de salud más complejas de las personas, por lo que es necesario que se apoyen y complementen por diferentes niveles de atención especializada, desde la consulta externa hasta el nivel hospitalario, interconectadas con las redes integradas de servicios de salud ${ }^{(4)}$. En ese sentido, los sistemas de atención de salud deben trabajar de forma integrada ${ }^{(5)}$, lo cual exige buenos sistemas de información que permitan «planificar, gestionar y evaluar el desempeño» ${ }^{(2)}$.

\section{ATENCIÓN PRIMARIA EN SALUD DIGITALIZADA}

La digitalización de la atención primaria en salud es un proceso complejo que requiere no sólo de desarrollo tecnológico, sino de un marco conceptual articulado con políticas, acciones y estrategias para implementar un sistema de información en salud integrado que permita que la información en salud sea lo más completa, eficiente, de calidad y que esté disponible oportunamente, para mejorar la calidad de vida de las personas y modernizar de manera significativa la salud pública ${ }^{(3)}$.

Una atención primaria en salud digitalizada requiere gobernanza, rectoría, óptima infraestructura e infoestructura, financiamiento apropiado, modelo de inversiones, y políticas públicas de gobierno $\mathrm{y}$, sobre todo, recursos humanos capacitados, motivados e innovadores, y capaces de enfrentar la gestión del cambio (3); asimismo, es importante entender la cultura organizacional y los factores sociotécnicos como desafíos del proceso de transformación digital. Es clave fortalecer las destrezas y conocimientos del personal de atención primaria, a través de programas de capacitación continua a nivel de pre y posgrado, para utilizar e incluso implementar adecuadamente los sistemas de información en salud. En lo posible, el personal de atención primaria debe estar involucrado desde las etapas iníciales del desarrollo de un sistema de información, para disminuir los riesgos de la resistencia al cambio ${ }^{(3)}$.

Un punto importante a destacar es que, para lograr una atención primaria digitalizada, se necesitan mejorar procesos, integrary evaluarnuevas yapropiadas tecnologías y desplegar una óptima infraestructura incluyendo su mantenimiento y monitoreo ${ }^{(3)}$. Implementar nuevas tecnologías incrementa las capacidades y fortalece la atención primaria en salud ${ }^{(6,7)}$.

\section{AVANCES EN LAS POLÍTICAS E INICIATIVAS RELACIONADAS A LA SALUD DIGITAL EN PERÚ}

En los últimos diez años, el Perú ha logrado avances claves en cuanto a políticas de salud digital, en la Tabla 1 se resume los principales documentos y se complementa con artículos publicados anteriormente que resumen normativas $y$ documentos legales relacionados a las políticas públicas del uso de las tecnologías de la información y la comunicación en salud en el Perú ${ }^{(8,9)}$.

El desarrollo de tecnologías y la implementación de sistemas de información robustos, eficientes, oportunos son componentes fundamentales de una estrategia para renovar y fortalecer la atención primaria en salud. Una buena gerencia en atención primaria puede generar ciertos cambios basándose en el uso de herramientas estratégicas como las historias clínicas electrónicas y la telesalud ${ }^{(10)}$.

\section{REGISTROS ELECTRÓNICOS EN SALUD Y LAS HISTORIAS CLÍNICAS ELECTRÓNICAS}

Un componente fundamental para fortalecer la atención primaria en salud es la implementación de los registros electrónicos en salud. En específico, la historia clínica electrónica es la «historia clínica registrada en forma unificada, personal, multimedia, refrendada con la firma digital del médico u otros profesionales de salud, cuyo tratamiento (registro, almacenamiento, actualización, acceso y uso) se realiza en estrictas condiciones de seguridad, integralidad, autenticidad, confidencialidad, exactitud, inteligibilidad, conservación, y disponibilidad a través de un Sistema de Información de Historias Clínicas Electrónicas, de conformidad con las normas aprobadas por el Ministerio de Salud, como órgano rector competente» ${ }^{(11)}$.

Avances importantes se han dado en establecimientos de EsSalud (modalidad asociación pública privada) que utilizan certificados digitales para la firma de las historias clínicas; asimismo, según el MINSA, existen más de 500 establecimientos de salud en los que se ha implementado la telemedicina y la historia clínica electrónica a diciembre del $2018^{(12)}$. Además, el MINSA ha implementado módulos electrónicos de inmunizaciones que incluye el carné de vacunación en 480 centros de salud a diciembre del $2018^{(12)}$. Esto permite la interconexión de establecimientos de menor complejidad con establecimientos de salud de mayor complejidad bajo un enfoque de redes integradas de salud. Asimismo, el seguimiento nominal de los ciudadanos es fundamental para brindar una atención más eficiente. Los registros electrónicos nominales facilitan no sólo la continuidad de la atención sino también la trazabilidad a la atención de los pacientes de los establecimientos de salud. 
Tabla 1. Principales documentos legales relacionados a las políticas públicas sobre salud digital en el Perú (2015-2017)

\begin{tabular}{|c|c|}
\hline Documento legal & Descripción \\
\hline $\begin{array}{l}\text { Resolución Ministerial N³89- } \\
\text { 2017/MINSA }\end{array}$ & $\begin{array}{l}\text { Aprueba a nivel nacional el «Padrón Nominal distrital de niñas y niños menores de seis (06) } \\
\text { años de edad». }\end{array}$ \\
\hline $\begin{array}{l}\text { Resolución Ministerial N } 120- \\
\text { 2017/MINSA }\end{array}$ & $\begin{array}{l}\text { Aprueba la Directiva Administrativa } N^{\circ} 230-M I N S A / 2017 / O G T I \text { - «Directiva Administrativa que } \\
\text { establece los estándares y criterios técnicos para el desarrollo de los sistemas de información } \\
\text { en salud». }\end{array}$ \\
\hline $\begin{array}{l}\text { Resolución Ministerial N } 119- \\
\text { 2017/MINSA }\end{array}$ & $\begin{array}{l}\text { Aprueba la Directiva Administrativa } N^{\circ} \text { 229-MINSA/2017/OGTI «Directiva Administrativa para } \\
\text { el Uso de Servicios Informáticos del Ministerio de Salud». }\end{array}$ \\
\hline $\begin{array}{l}\text { Resolución Ministerial Nº 0214- } \\
\text { 2017/MINSA }\end{array}$ & Aprueba la Guía Técnica para el correcto llenado del Certificado de Defunción. \\
\hline $\begin{array}{l}\text { Resolución Ministerial Nº 074- } \\
\text { 2017/MINSA }\end{array}$ & $\begin{array}{l}\text { Aprueba D.A. N N 227-MINSA/2017/OGTI - Directiva Administrativa de Organización del } \\
\text { Sistema de Gestión de Seguridad de la Información del Ministerio de Salud. }\end{array}$ \\
\hline $\begin{array}{l}\text { Resolución Ministerial Nº 058- } \\
\text { 2017/MINSA }\end{array}$ & 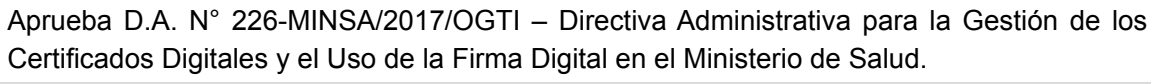 \\
\hline $\begin{array}{l}\text { Resolución Ministerial Nº } 021- \\
\text { 2017/MINSA }\end{array}$ & 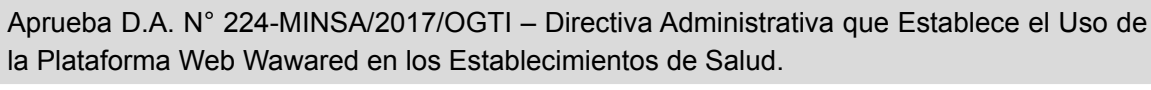 \\
\hline Decreto Legislativo $N^{\circ} 1303$ & Dicta disposiciones destinadas a optimizar los procesos vinculados a Telesalud \\
\hline Decreto Legislativo $N^{\circ} 1306$ & $\begin{array}{l}\text { Dicta disposiciones destinadas a optimizar los procesos vinculados al Registro Nacional de } \\
\text { Historias Clínicas Electrónicas, creado según Ley } N^{\circ} 30024 \text {, del 22-05-2013. }\end{array}$ \\
\hline $\begin{array}{l}\text { Resolución Ministerial Nº 978- } \\
\text { 2016/MINSA }\end{array}$ & $\begin{array}{l}\text { Aprueba D.A. N 221-MINSA/2016/OGTI - Directiva Administrativa que Autoriza el Uso de la } \\
\text { Firma Digital en los Actos Médicos y Actos de Salud. }\end{array}$ \\
\hline $\begin{array}{l}\text { Resolución Ministerial N² 280- } \\
\text { 2016/MINSA }\end{array}$ & $\begin{array}{l}\text { Aprueba D.A. } \mathrm{N}^{\circ} \text { 216-MINSA/OGTI-V.01 - Directiva Administrativa que Establece el } \\
\text { Procedimiento para la Certificación de las Defunciones, y deja sin efecto la R.M. N }{ }^{\circ} 332- \\
\text { 2005/MINSA. }\end{array}$ \\
\hline Ley $N^{\circ} 30421$ & Ley Marco de Telesalud \\
\hline R.M. N 004-2016-PCM & $\begin{array}{l}\text { Aprueban uso obligatorio de la Norma Técnica Peruana «NTP ISO/IEC 27001:2014 - } \\
\text { Tecnología de la Información. Técnicas de Seguridad. Sistemas de Gestión de Seguridad } \\
\text { de la Información. Requisitos. } 2^{\text {a }} \text {. Edición», en todas las entidades integrantes del Sistema } \\
\text { Nacional de Informática. }\end{array}$ \\
\hline R.M. N 004-2016-PCM & $\begin{array}{l}\text { Aprueban uso obligatorio de la Norma Técnica Peruana «NTP ISO/IEC 27001:2014 - } \\
\text { Tecnología de la Información. Técnicas de Seguridad. Sistemas de Gestión de Seguridad de } \\
\text { la Información, en todas las entidades del Sistema Nacional de Informática». }\end{array}$ \\
\hline D.S. N 039-2015-SA & $\begin{array}{l}\text { Aprueba el Reglamento de la Ley } N^{\circ} 30024 \text { - Ley que Crea el Registro Nacional de Historias } \\
\text { Clínicas Electrónicas. }\end{array}$ \\
\hline $\begin{array}{l}\text { Resolución Ministerial N } 431 \text { - } \\
\text { 2015/MINSA }\end{array}$ & $\begin{array}{l}\text { Aprueba el Documento Técnico «Política de Seguridad de la Información del Ministerio de } \\
\text { Salud - MINSA». }\end{array}$ \\
\hline $\begin{array}{l}\text { Resolución Ministerial N³50- } \\
\text { 2015/MINSA }\end{array}$ & $\begin{array}{l}\text { Crea el Repositorio Único Nacional de Información en Salud - REUNIS, y dispone que los } \\
\text { organismos públicos adscritos al Ministerio de Salud, proporcionen información necesaria. }\end{array}$ \\
\hline $\begin{array}{l}\text { Resolución Ministerial N 096- } \\
\text { 2015/MINSA }\end{array}$ & $\begin{array}{l}\text { Aprueba a nivel nacional el Padrón Nominal Distrital de Niños y Niñas Menores de Seis (6) } \\
\text { Años de Edad. }\end{array}$ \\
\hline
\end{tabular}

\section{INTEGRANDO LA ATENCIÓN PRIMARIA CON SERVICIOS DE TELESALUD}

La telesalud es una importante herramienta tecnológica que brinda mayor accesibilidad al personal asistencial de salud, en especial en áreas rurales, zonas aisladas y con limitada capacidad resolutiva a través de la conexión con establecimientos de salud ${ }^{(13)}$. La telesalud es un potente instrumento que posibilita no sólo las atenciones a larga distancia en los establecimientos de salud de atención primaria, sino que también a través de la telesalud podemos optimizar la brecha de especialistas en el Perú. Además, optimiza la coordinación de la gestión de los establecimientos de salud de atención primaria, acelerando decisiones y favoreciendo la desconcentración. Según el MINSA, a finales de noviembre del 2018, más 
de 7,200 telemamografías se realizaron en el Hospital de Emergencias Villa El Salvador como Centro Nacional Consultor de Teleimágenes, brindando apoyo al diagnóstico a distancia del cáncer de mama en 17 hospitales de 12 regiones del país ${ }^{(14)}$. La región con mayor demanda de este método diagnóstico es Junín, mediante la conexión que se tiene con los hospitales de Chanchamayo y Jauja, a través de la Red Nacional de Telesalud ${ }^{(14)}$.

La interconexión de establecimientos de salud de primer nivel de atención con hospitales de mayor complejidad a través de servicios de telesalud es una estrategia clave para fortalecer los sistemas de salud ${ }^{(7)}$

Por otro lado, a través del Centro Nacional de Telemedicina (CENATE) deEsSalud, la telemedicina se viene implementando desde setiembre del 2013. A junio del 2017, se efectuaron más de 118 mil estudios de imágenes, entre radiografías, mamografías y tomografías. Además, se realizaron más de 3200 teleconsultas ${ }^{(15)}$ en los establecimientos de salud de EsSalud. La interconexión de los establecimientos de salud de EsSalud ha permitido solucionar problemas de salud a nivel local, lo que ha generado un ahorro de más de seis millones de soles en el traslado de pacientes.

\section{TELEGESTIÓN PARA UNA EFICIENTE}

Diversas estrategias se vienen implementando para optimizar la coordinación entre las autoridades nacionales y regionales por medio de la telegestión ${ }^{(16)}$ o telereuniones ejecutivas ${ }^{(17)}$ definidas como "reuniones de trabajo de ejecutivos, jefes de servicios clínicos, especialistas y departamentos administrativos de las redes de salud». En el Ministerio de Salud del Perú, es usual la conexión en simultáneo de las 25 Direcciones y Gerencias Regionales de Salud para coordinaciones sobre campañas sanitarias, para temas sanitarios y epidemiológicos e incluso para las presentaciones de las salas situacionales virtuales, espacios dinámicos para conocer la situación de salud de las enfermedades o daños, con los establecimientos de salud de atención primaria.

La telegestión permite coordinaciones permanentes para tomar decisiones en tiempo real desde el nivel central con las regiones. Además de las videoconferencias en tiempo real, se complementa las acciones de monitoreo y gestión con el uso de la aplicación Whatsapp, que no sólo permiten el intercambio de información sino también se pueden convertir en una herramienta de respaldo y motivación. Estas herramientas de relativo bajo costo vienen siendo utilizadas con éxito para la gestión en salud en otros países en vías de desarrollo ${ }^{(18)}$.

\section{PERSPECTIVAS A FUTURO PARA DESARROLLAR UNA ATENCIÓN PRIMARIA EN SALUD DIGITALIZADA}

Existen muchos retos por superar para lograr una atención primaria en salud digitalizada en el Perú, las cuales se han descrito previamente en la literatura nacional $(9,13,19)$ e internacional ${ }^{(20,21)}$. En primer lugar, es necesario desplegar la implementación del Registro Nacional de Historias Clínicas Electrónicas con el objetivo de integrar la diversidad de historias clínicas electrónicas en el Perú, entre establecimientos de salud de atención primaria con hospitales de mayor complejidad. Esto permitirá mejorar la calidad y continuidad de la atención de salud. Para lograr este esfuerzo, es importante implementar diversos registros electrónicos en salud a nivel nacional. La Superintendencia Nacional de Salud cuenta con registros electrónicos claves para la gestión de información en atención primaria y deben ser fortalecidos ${ }^{(22)}$.

Por otro lado, es clave fortalecer el Repositorio Único Nacional de Información en Salud, aprobado mediante Resolución Ministerial $N^{\circ} 350-2015 /$ MINSA, que permita explotar al máximo la enorme cantidad de datos que maneja el Ministerio de Salud a través de los establecimientos de salud de atención primaria y los de mayor complejidad resolutiva, e implementar diversas estrategias de visualización de datos por un lado, y dar valor agregado a la gestión de los datos con herramientas de "data mining» e incluso implementar algoritmos de predicción y herramientas de inteligencia artificial para beneficio y optimización de diversos procesos en atención primaria en salud ${ }^{(23)}$.

El ciudadano digital hoy cuenta con diversas herramientas digitales con enorme potencial para mejorar el cuidado de su salud. Los dispositivos móviles a través del uso de celulares o sensores portátiles permiten monitorizar signos y alertar condiciones que requieren atención ${ }^{(7)}$. Por otro lado, los portales electrónicos del paciente, o portales de salud del ciudadano son iniciativas implementadas en muchos países ${ }^{(7,24)}$ y que se deberían implementar a la brevedad en el Perú para brindar directamente servicios en línea a la población en general. Este portal debe ofrecer también información en salud de utilidad no sólo para los pacientes sino para el público en general. En muchos países los portales personales de salud se complementan de manera muy eficiente con las historias clínicas electrónicas ${ }^{(24) .}$

La interoperabilidad es un aspecto crítico que tiene que ser intensamente trabajado a través de una plataforma eficiente de intercambio de información del sector salud, que optimice el intercambio de información en el sector salud y con las entidades del Estado. Para ello, es clave culminar de implementar los catálogos sectoriales en salud, para el registro e intercambio estandarizado de información, en cumplimento de la identificación estándar de datos 
en salud ${ }^{(8)}$. Finalmente, los sistemas de hechos vitales, incluyendo el Certificado de Nacido Vivo en línea ${ }^{(25)}$, y el Certificado de Defunciones en línea a través del Sistema Informático Nacional de Defunciones - SINADEF (26), deben ser implementados en todos los establecimientos de salud que sean necesariosn para optimizar los procesos de los registros de hechos vitales y los registros civiles. Estos registros electrónicos claves sientan las bases para integrar con otros sistemas de información para la atención primaria en salud, incluyendo la telesalud (27).

\section{CONCLUSIÓN}

Se han revisado las principales políticas en salud digital y las principales iniciativas implementadas en el Perú. Es necesario establecer un gobierno electrónico en salud con sistemas de información sectoriales, nominales que permitan interconectar los establecimientos de salud de primer nivel de atención con establecimientos de mayor complejidad. Asimismo, es necesario fortalecer el primer nivel de atención y capacitar a los profesionales de salud de la atención primaria de manera continua, incluyendo las herramientas de telecapacitación y potenciar las redes de investigación y educación en telesalud ${ }^{(28)}$. Es fundamental implementar sistemas integrados incluyendo el registro nacional de historias clínicas electrónicas y continuar expandiendo los registros electrónicos ${ }^{(29)}$ y los servicios de telesalud que incrementarán la calidad de la atención, así como la continuidad en el cuidado de la persona. Todo ello contribuirá a desarrollar una atención primaria en salud digitalizada y permitirá que el ciudadano digital, aquel que utiliza internet regular y eficazmente de forma cotidiana, no sólo disponga de mayor información ${ }^{(30)}$, sino que sea capaz de evaluar de manera eficaz y eficiente la información sobre salud que encuentra en internet, y que también tenga la capacidad de participar e interactuar en una sociedad conectada (online) y de ejercer mayor control sobre las decisiones tomadas sobre su salud. Hoy el ciudadano digital ha evolucionado de ser protagonista de plataformas web básicas a plataformas que embeben lenguajes con inteligencia artificial ${ }^{(31)}$, donde no solamente el ciudadano es un receptor y creador de contenidos en salud sino un prosumidor, es decir, receptor, creador y «resignificador» de la comunicación, todo al mismo tiempo ${ }^{(32)}$.

Los autores rinden homenaje al doctor David Tejada de Rivero (1929 - 2018), quien fuera Sub Director General de la Organización Mundial de la Salud y coordinador general de la Conferencia Internacional sobre Atención Primaria de Salud de Alma-Ata celebrada en Kazajistán en 1978, dos veces Ministro de Salud del Perú (1974 y 1985), y el único peruano condecorado por sus invaluables contribuciones a la mejora de salud y el bienestar de los pueblos como "Héroe de la Salud Pública de las Américas" por la Organización Panamericana de la Salud; quien planteó el verdadero espíritu, contenido y esencia de Alma-Ata: "el cuidado integral de la salud para todos y por todos" (33).

Contribución de los autores: WHC, MHS y EE participaron en la redacción del manuscrito y aprobaron la versión final a publicar.

Fuentes de financiamiento: autofinanciado.

Conflicto de interés: Los autores declaran no tener conflictos de interés en la publicación de este artículo.

\section{REFERENCIAS BIBLIOGRÁFICAS}

1. Osorio AG, Álvarez CV. La Atención Primaria de Salud: desafíos para su implementación en América Latina. Aten Primaria. 2013;45(7):384-92.

2. Macinko J, Montenegro $\mathrm{H}$, Nebot Adell C, Etienne C y Grupo de Trabajo de Atención Primaria de Salud de la Organización Panamericana de la Salud. La renovación de la atención primaria de salud en las Américas. Rev Panam Salud Publica. 2007; 21(2/3):73-84

3. OMS. Informe sobre la salud en el mundo 2008: La atención primaria de salud más necesaria que nunca. Ginebra: OMS; 2008.

4. Ocampo-Rodríguez MV, BetancourtUrrutia VF, Montoya-Rojas JP, BautistaBotton DC. Sistemas y modelos de salud, su incidencia en las redes integradas de servicios de salud. Gerencia y Políticas de Salud. 2013; 12(24):114-129.
5. Starfield B. Atención Primaria: Equilibrio entre necesidades de salud, servicios y tecnología. 2da edición. Madrid: Masson; 2002.

6. Alfaro M, Bonis J, Bravo R, Fluiters E, Minué S. Nuevas tecnologías en atención primaria: personas, máquinas, historias y redes. Informe sespas 2012. Gac Sanit. 2012;26 Suppl 1:107-12.

7. Young HM, Nesbitt TS. Increasing the capacity of primary care through enabling technology. J Gen Intern Med. 2017;32:398-403.

8. Curioso WH, Espinoza-Portilla E. Marco conceptual para el fortalecimiento de los Sistemas de Informacion en Salud en el Peru. Rev Peru Med Exp Salud Publica. 2015;32(2):335-42.

9. Curioso WH. E-Salud en Perú: implementación de políticas para el forta- lecimiento de sistemas de información en salud. Rev Panam Salud Publica. 2014;35(5/6):437-41.

10. Bodenheimer T, Grumbach K. Electronic technology: a spark to revitalize primary care? JAMA 2003;290: 259-64.

11. Decreto Legislativo 1306. Decreto Legislativo que Optimiza procesos Vinculados al Registro Nacional de Historias Clínicas Electrónicas. (30 de diciembre 2016).

12. Ministerio de Salud. Estamos ingresando a la transformación digital en salud. Disponible en: https:/www.gob.pe/ institucion/minsa/noticias/23383ministra-pessah-estamos-ingresando-a-latransformacion-digital-en-salud

13. Curioso WH. La telesalud y las nuevas fronteras de la informática biomédica en el Perú. Rev Peru Med Exp Salud Publica 2015;32(2):217-20. 
14. Ministerio de Salud. Hospital de Villa El Salvador realizó más de 7,200 telemamografias. Disponible en: https:// www.gob.pe/institucion/minsa/noticias/23215-hospital-de-villa-el-salvador-realizo-mas-de-7-200-telemamografias

15. EsSalud. Presidente de EsSalud inauguró Primer Congreso Internacional de Telemedicina. 19 Octubre 2017. Disponible en: http://www.essalud.gob.pe/presidente-de-essalud-inauguro-primer-congreso-internacional-de-telemedicina/

16. Ministerio de Salud del Perú. Instituto Nacional de Salud el Niño - San Borja. Manual de Gestión de Procesos y Procedimientos. Gestión del Desarrollo dela Atención Especializada a las Regiones del Instituto Nacional de Salud del NiñoSan Borja. Resolución Directoral No $46-$ 2017/INSN-SBA. (8 de marzo 2017).

17. Decreto Supremo No 028-2005-MTC. Plan Nacional de Telesalud. (22 de octubre 2005).

18. Todd CS, Mills SJ, Innes AL. Electronic health, telemedicine, and new paradigms for training and care. Curr Opin HIV AIDS. 2017;12(5):475-487.

19. Henríquez-Suarez M, Becerra-Vera CE, Laos-Fernández EL, EspinozaPortilla E. Evaluación de programas de salud electrónica en el Perú: enfoque multidisciplinario y perspectivas actuales. Rev Peru Med Exp Salud Publica. 2017;34(4):731-7.

20. Baird A, Nowak S. Why primary care practices should become digital health information hubs for their patients. BMC Fam Pract. 2014 Nov 25;15:190.

21. de Lusignan $S$. What is primary care informatics? J Am Med Inform Assoc. 2003;10(4):304-9.
22. OECD. Monitoring Health System Performance in Peru. Data and Statistics. 2017. Disponible en: http://www. oecd.org/countries/peru/monitoringhealth-system-performance-in-peru9789264282988-en.htm

23. Bates DW, Saria S, Ohno-Machado L, Shah A, Escobar G. Big Data In health care: Using analytics to identify and manage high-risk and high-cost patients. Health Aff (Millwood). 2014;33:1123-31.

24. Gil-Quevedo W, Agurto-Távara E, Espinoza-Portilla E. Ciudadanos informados y empoderados: claves para el pleno ejercicio de los derechos en salud. Rev Peru Med Exp Salud Publica. 2017;34(2):311-315.

25. Curioso WH, Pardo K, Loayza M. Transformando el sistema de información de nacimientos en el Perú. Rev Peru Med Exp Salud Publica. 2013;30(2):303-7.

26. Miki J, Rampatige R, Richards N, Adair T, Cortez-Escalante J, Vargas-Herrera J. Saving lives through certifying deaths: assessing the impact of two interventions to improve cause of death data in Perú. BMC Public Health. 2018;18(1):1329. doi: 10.1186/s12889-018-6264-1.

27. Curioso WH, Espinoza-Portilla E. Políticas E Iniciativas De E-Saúde Para O Fortalecimento De Sistemas De Informação Em Saúde No Peru. En: Pesquisa sobre o uso das tecnologias de informação e comunicação nos estabelecimentos de saúde brasileiros: TIC Saúde 2016 = Survey on the use of information and communication technologies in Brazilian healthcare facilities : ICT in health 2016 [libro electrónico] / Núcleo de Informação e Coordenação do Ponto BR [editor] - São Paulo : Comitê Gestor da Internet no Brasil, 2017.
28. Curioso Vílchez W. Red Nacional de Investigación y Educación (RNIE): Avances y perspectivas de la Telesalud en Perú. I Congreso Iberoamericano de Telesalud y Telemedicina; 2014 Octubre 16-18; Lima, Perú.

29. Trumbo SP, Contreras M, García AGF, Díaz FAE, Gómez M, Carrión V, Ruiz KJP, Aquije R, Danovaro-Holliday MC, Velandia-González M. Improving immunization data quality in Peru and Mexico: Two case studies highlighting challenges and lessons learned. Vaccine. 2018;36(50):7674-7681.

30. Pineda M. Nuevas formas de ciudadanía asociadas a las redes de comunicación globales: el ciudadano digital. Historia Actual Online, 2011;24:163-183.

31. Sepúlveda-Cardona EA, Restrepo-Escobar SM. e-Salud y el estado de las Apps para adicciones: un análisis articulado de los cibermedios para ecosistemas digitales pensados a la Atención Primaria en Salud. Health and Addictions. 2018; 18(2):185- 194.

32. Álvarez-Moreno MA, Botero-Montoya LH. De ciudadanos a prosumidores nuevas representaciones y consumo. Anagramas -Rumbos y sentidos de la comunicación-2015;13(26):11-15.

33. Tejada de Rivero DA. Lo que es la atención primaria de la salud: algunas consideraciones a casi treinta y cinco años de Alma-Ata. Rev Peru Med Exp Salud Publica. 2013;30(2):283-7.

Correspondencia: Walter H. Curioso. Dirección: Department of Biomedical Informatics and Medical Education, School of Medicine, University of Washington. Seattle, Washington, EE. UU..

Correo electrónico: wcurioso@uw.edu; waltercurioso@gmail.com

\section{Nuestros artículos se encuentran indizados en:}

$$
\text { Publared }
$$

www.pubmed.gov 